\title{
Sensing-time optimization in Cognitive Radio enabling Smart Grid
}

\author{
A.S. Cacciapuoti, M. Caleffi, F. Marino and L. Paura \\ Dep. of Electrical Engineering and Information Technology (DIETI) \\ University of Naples Federico II \\ Naples, Italy \\ Email: \{ angelasara.cacciapuoti, marcello.caleffi, francesco.marino, paura \}@unina.it
}

\begin{abstract}
The paper deals with the reduction of the channel outage in a smart grid scenario since it plays a crucial role on the control performance of the Demand/Response Management. A study on a two-way cognitive-based switching procedure is carried out and tools for the optimum sensing-time evaluation are provided. Such evaluation considers a cost function that takes into account both the sensing-accuracy improvement gained by increasing the sensing time and the transmission-capacity degradation induced by sensing-time increasing.
\end{abstract}

Keywords—smart grid, cognitive radio; channel outage

\section{INTRODUCTION}

Demand/Response Management (DRM) is, as well-known, a key functionality in the energy management of a next generation smart grid since it can significantly reduce the peak power consumption. Very recently, great attention [1] has been devoted towards real-time interactions between consumers and provider by introducing smart meters in the grid. In fact, smart meters aim at optimizing the use of the available power by accounting for the energy market prices of the different power sources. The exchange of information among the smart grid nodes must be performed for many data categories in real-time and, therefore, this requires a reliable and efficient communication network that, moreover, must be realized at an affordable cost. The wireless technology can be able to offer interesting solutions thanks to the undeniable vantages that such a technology can assure such as flexibility, low cost, high speed of installation and return of investment in short time. On the other hand it is also well-known how hostile is the wireless media and, consequently, innovative transmission solutions have to be put in place to guarantee the satisfactory level of reliability, low temporal latency, high data rates and power as well as spectrum efficiency.

This work was partially supported by the Italian government under PON0102425 "SIRIO: Services for wIreless netwoRk Infrastructure beyOnd 3G", and under PON01-00744 "DRIVEIN2: DRIVEr Monitoring: Technologies, Methodologies, and IN-vehicle INnovative systems for a safe and ecocompatible driving".
The smart grid is commonly deployed in a wide geographical region [2] and, consequently, the communication infrastructure is envisioned to be a multilayer topology that extends across the whole smart grid from the home area networks (HAN) to the neighbourhood area networks (NAN) and wide area networks (Fig.1). More specifically, in HAN various smart meters from home are connected to the gateway by utilizing wireless protocols and technologies such as ZigBee, Bluetooth, etc.,. The NAN connects multiple HAN gateways to a local access point and, finally, WAN provide communication paths among the NAN access points and the utility systems to exchange information. This three-layered communication structure has to deal with several important and challenging issues such as:

i. Tremendous amount of heterogeneous data (greater than tens of thousands of Tera bytes in 2015);

ii. Integration of the power sources - power from utilities and power from different distribute renewable energy sources (e.g. , wind and solar sources) present in the NAN; such integration is mandatory if providers as well as consumers want to take advantage for the different prices and availability of the power sources;

iii. Highly varying traffic - the smart grid has to deal with a huge amount of data (both real-data and archival data) and the amount of data-exchange highly varies during the day so that the communication infrastructure requires both high data rates, low temporal latency to assure real-time services and, of course a satisfactory level of reliability;

iv. Inter-operability - due to the multi-layered topology of the network a multiplicity of technologies and standards are utilized which, require to solve many and important issues of interoperability;

v. Quality of Service (QoS) - unfortunately the large amount of data has also characterized by a large variety of categories each of one imposes different transmission (bandwidth, latency, reliability etc.) and security requirements.

vi. Security - the wireless technology exposes, farther than the fixed networks, smart grids to outside attacks. Therefore, the security has to be deeply studied because there are many threats within utilities, such as, for 
example, indiscretions by employees and authorization violations [3].

The previous considerations regarding the requirements of the communication infrastructure for smart grid evidence the need to find out effective solutions to the lack of spectral resources, mainly due to a very inefficient way to utilize such precious resources [4]. The spectrum inefficiency is mainly due to the static spectrum access, and, for this reason the FCC has encouraged the utilization and development of the cognitive paradigm [5] for the dynamic spectrum access (DSA) [6] and [7]. According to the cognitive radio (CR) paradigm, secondary users (SU's), referred to also cognitive users, sense the channel looking for spectral holes in the licensed bandwidth. If such holes are discovered available they can use them, provided that they do not interfere with the licensed users referred in the following to the primary users (PU). Very recently, the extensive use of cognitive paradigm has been proposed [3] for a smart grid: it is suggested to apply the cognitive paradigm for all three sub-networks constituting the smart grid communication infrastructure, namely, the HAN, the NAN and the WAN. Since the channel outage significantly affects the control performance of the DRM, very recently attention [8] to reduce such outage has been devoted by exploiting the channel resources that the cognitive paradigm can make available in the HAN sub-network.

To reduce the outage channel in the HAN and NAN subnetworks, where the radio cognitive paradigm seems to be more promising, we propose to utilize the same switching cognitive-based procedure of [8] which employs a two-way communication.

Unlike [8], this paper focus on the sensing-time optimization by accounting for the fact that the sensing time and the transmission time take turns and, that the sensing period, namely their summation, is limited since the regulatory rules impose a continuous and adequate updating of the channelstate (busy or idle from PU). Such a sensing period is assessed to assure the interference requirements and its value depends also by the PU traffic activity statistics. Therefore, if the sensing time is increased to reduce the sensing errors (falsealarm probability and detection probability) the time dedicated for the transmission become smaller. From such a consideration it results that a trade-off between the sensing accuracy, and consequently channel outage, and transmission capacity must be taken into account.

The present paper considers such an issue and proposes how to assess the sensing time in order to optimize jointly the sensing accuracy (namely, channel outage) and the transmission capacity. Specifically, a cost function which accounts for the channel outage and the transmission capacity, is proposed and maximized with respect to the sensing time.

The paper is organized as follows: Section II synthesizes the related papers whereas Section III presents the time-sensing optimization procedure. Section IV provides numerical results and Section V is devoted to the conclusions and final remarks.

\section{RELATED PAPER}

In [9] the authors propose a CR channel allocation and traffic scheduling scheme which accounts for the channel switching as well as for the spectrum sensing errors and solve a systemutility optimization problem for a smart communication system. The paper focus on wireless sensor networks, namely a HAN sub-network, to perform an intelligent monitoring for a smart grid scenario and categorizes the multimedia traffic to prioritize it in order to optimize the scheduling with reference to a single-hop CR network system. The rule of the sensing time is not considered as well as the fact that the infrastructure is a three-layered network. In [8] the authors with reference to HAN, analyse the impact of the channel outage on the DRM control performances. It, as well-known, reduces the profit of the power provider as well as the social welfare of the smart grid. They propose a cognitive-based two-way communication switching procedure to reduce the channel outage and study the trade-off between better control performance and higher communication cost which both increase by increasing the sensing time. In the paper it is shown that there exists a unique optimal sensing time which assures the optimum trade-off under the assumption that energy detectors are employed for the spectrum sensing by the consumers and assuming that the signal-to-noise ratios at the transceiver are equal each other regardless of the different locations of the nodes of smart grid. More specifically, the SG nodes are assumed to have the same spectrum sensing capabilities, which is not the case in a real system. The channel-outage influence on the DRM control performance is also analysed in [1]. In order to lower the channel outage not only the temporal opportunities originated by the absence of PU activity in its protection range ${ }^{1}$ [10-13] but also the spatial opportunities caused also, for example, by the relative mobility between PU and SU [12] can be exploited. CU's can be out of the protection range and, therefore spatial spectrum opportunities can occur, allowing to lower the channel outage. A joint temporal and spatial spectrum sharing scheme is proposed in [1] and the results of the performance analysis show that the joint spatial and temporal spectrum sharing can increase the spectrum SG opportunities and lower the outage probability and, consequently, improve the DRM control performances provided that the primary user transmission power is not too large.

${ }^{1}$ The protection range is determined by the PU transmission range and the $\mathrm{CU}$ interference range. 


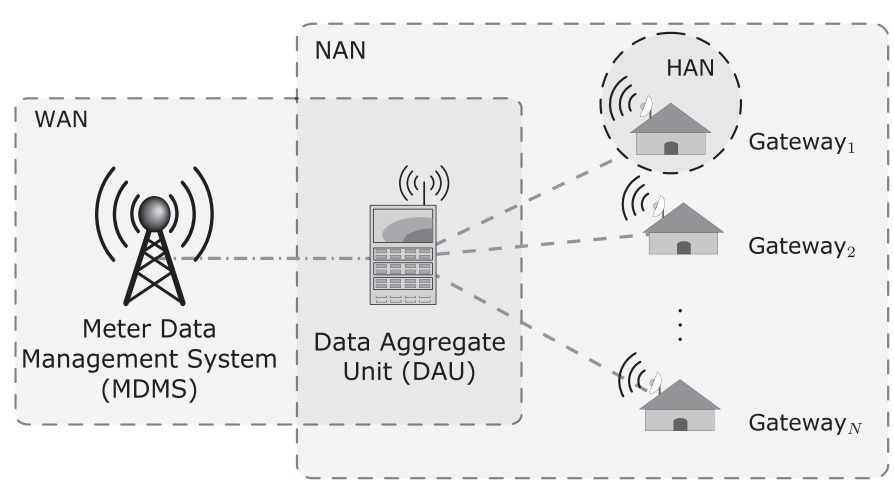

Fig. 1 System Model

\section{THE PROPOSED OPTIMIZATION PROCEDURE}

The smart grid under consideration consists of one power provider and $\mathrm{N}$ power consumers, which deliver/receive their data towards/from the Demand/ Response Management (DRM). Each consumer collects the data periodically delivered by its meters or to be delivered towards its meters at a home gateway utilizing a home area network (HAN). Each gateway adopts a two-way communication cognitive-based procedure to select between a channel belonging to a primary network, referred in the following as cognitive channel, say $\mathrm{Ch}_{\mathrm{c}}$ (i) $(\mathrm{i}=1,2 \ldots, \mathrm{N})$ and a channel which utilizes the unlicensed spectrum, referred in the following as unlicensed channel, say $\mathrm{Ch}_{\mathrm{ul}}(\mathrm{i})$. More precisely, the ith consumer chooses its channel according to the following rule: if the spectrum sensing procedure detects the $\mathrm{Ch}_{\mathrm{c}}(\mathrm{i})$ idle, the smart gateway switches the data transmission to $\mathrm{Ch}_{\mathrm{c}}(\mathrm{i})$. Such an event occurs with probability $\mathrm{P}_{\mathrm{sw}}(\mathrm{i}) \mathrm{P}_{\mathrm{og}}(\mathrm{i})$ where $\mathrm{P}_{\mathrm{sw}}(\mathrm{i})$ and $\mathrm{P}_{\mathrm{og}}(\mathrm{i})$ are defined as follows:

i) $P_{\mathrm{sw}}(\mathrm{i})$ is the probability that the switching occurs, namely the sensing detects the cognitive channel idle, namely it detects that the primary user does not utilize the channel $\mathrm{Ch}_{\mathrm{c}}(\mathrm{i})$ : this probability can be evaluated considering two possible mutually exclusive events: a) the primary channel is busy, which happens with probability $\mathrm{P}_{1}(\mathrm{i})^{2}$, and the sensing fails; b) the primary channel is idle which occurs with probability $1-\mathrm{P}_{1}(\mathrm{i})$ $=\mathrm{P}_{0}(\mathrm{i})$ and the sensing detects correctly that the channel is idle. Therefore:

$$
\begin{aligned}
& P_{s w}\left(\tau_{i}\right)= \\
& \quad=P_{1}(i)\left(1-P_{d}\left(\tau_{i}\right)\right)+ \\
& \quad+P_{0}(i)\left(1-P_{f}\left(\tau_{i}\right)\right)
\end{aligned}
$$

In (2.1) $\mathrm{P}_{\mathrm{d}}\left(\tau_{i}\right)$ and $\mathrm{P}_{\mathrm{f}}\left(\tau_{i}\right)$ are the detection probability and the false-alarm probability, respectively. The

2 The dependence on the index i accounts for the potential different PU network scenarios due to the different locations of the consumer gateways. Moreover, the assumption of statistical independence among the overall channel outages $P_{o g}\left(\tau_{i}\right)(\mathrm{i}=1,2, \ldots \mathrm{N})$ require the statistical independence among the switching events and the statistical independence among the unlicensed channel outages as well as the licensed ones. Such properties can be assured provided that the channels are appropriately assigned to each consumer. former is the probability that the channel is detected busy by the sensing procedure when the PU is in operation, whereas the latter is the probability that the channel is sensed busy when the PU is not in operation.

ii) $\quad \mathrm{P}_{\mathrm{og}}\left(\tau_{i}\right)$ is the channel-outage probability which refers to the channel utilized to deliver the data from the ith consumer gateway towards the DRM. It is equal to channel-outage probability of the $\mathrm{Ch}_{\mathrm{ul}}(\mathrm{i})$ if the cognitive-channel switching is not utilized whereas if the cognitive-based switching is adopted, the channeloutage probability is evaluated by considering the two mutually exclusive events:

a) no switching occurs and the unlicensed channel outage $\mathrm{Ch}_{\mathrm{ul}}(\mathrm{i})$ happens whose probability is given by $\left(1-\mathrm{P}_{\mathrm{sw}}\left(\tau_{i}\right)\right) \mathrm{P}\left(\mathrm{Ch}_{\mathrm{ul}}(\mathrm{i})\right)$;

b) the switching occurs and the cognitive channel outage $\mathrm{Ch}_{\mathrm{c}}$ (i) happens whose probability is given by $\left.\mathrm{P}_{\mathrm{sw}}\left(\tau_{i}\right)\right) \mathrm{P}\left(\mathrm{Ch}_{\mathrm{c}}(\mathrm{i})\right)$.

Therefore the channel-outage probability based on the adopted switching procedure is:

$$
\begin{aligned}
P_{o g}\left(\tau_{i}\right) & =\left(1-P_{S W}\left(\tau_{i}\right)\right) P\left(C h_{u l}(i)\right)+ \\
& +P_{S W}\left(\tau_{i}\right) P\left(C h_{c}(i)\right)= \\
& =\left[P\left(C h_{c}(i)\right)-P\left(C h_{u l}(i)\right)\right] \cdot P_{S W}\left(\tau_{i}\right)+ \\
& +P\left(C h_{u l}(i)\right)
\end{aligned}
$$

It is reasonable to assume that the quality of the licensed channel is better than the one of the unlicensed channel and, namely $P\left(C h_{c}(i)\right)<P\left(C h_{u l}(i)\right)$, therefore, it results from (2.2) that the two-way cognitive-based switching procedure assures a lower channel outage, namely, $\mathrm{P}_{\text {og }}\left(\tau_{i}\right)<\mathrm{P}_{\text {og ul }}(\mathrm{i})$. Moreover, the greater is the $\mathrm{P}_{\mathrm{sw}}\left(\tau_{i}\right)$ the lower is the channeloutage probability.

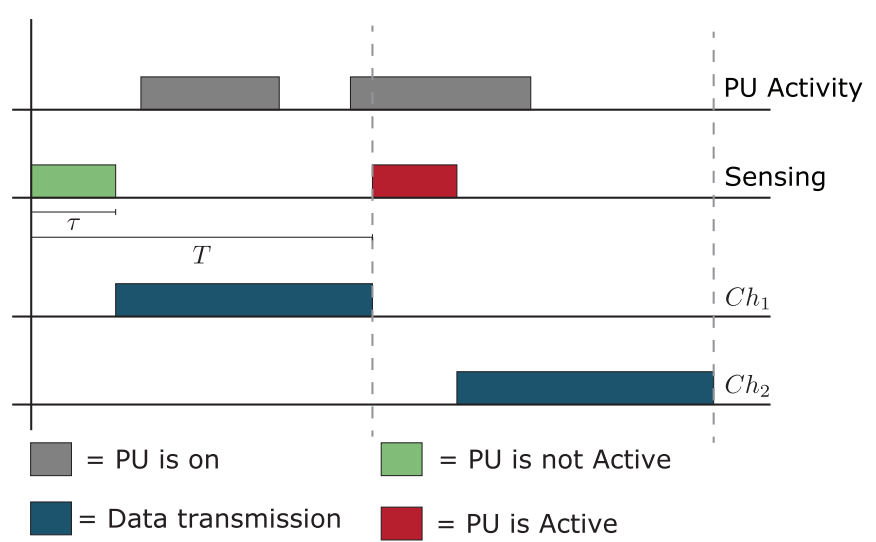

Fig. 2 Cognitive-Radio Framework for Two-way Communications.

Fig. 2 shows the cognitive-radio framework for the two-way communication structure: every $\mathrm{T}$ second each consumer performs a sensing whose duration is $\tau_{i}$ after that it starts to transmit for a time $\mathrm{T}-\tau_{\mathrm{i}}$ over the unlicensed channel $\mathrm{Ch}_{\mathrm{ul}}(\mathrm{i})$ if the sensing detects the cognitive channel busy, otherwise it 
delivers its data over the cognitive channel $\mathrm{Ch}_{\mathrm{c}}(\mathrm{i})$. There is no collision and no lost of opportunities if the sensing is errorfree. The switching probability, according to (2.1) depends on the PU traffic, namely by $\mathrm{P}_{1}\left(\tau_{i}\right)$ and by the sensing accuracy which, in turn, depends on both the detection probability $\mathrm{P}_{\mathrm{d}}\left(\tau_{i}\right)$ and the false-alarm probability $\mathrm{P}_{\mathrm{f}}\left(\tau_{i}\right)$. The former is not under the control of the sensing procedure whereas, the latter, by means $\mathrm{P}_{\mathrm{d}}\left(\tau_{i}\right)$ and $\mathrm{P}_{\mathrm{f}}\left(\tau_{i}\right)$ can be adequately optimized since they both depend on the sensing time $\tau_{\mathrm{i}}$.

Let us underline that $1-\mathrm{P}_{\mathrm{d}}\left(\tau_{i}\right)$ gives a measure of the interference amount caused by the $i$-th secondary user on the PU transmissions, and therefore the standards impose that:

$$
P_{d}\left(\tau_{i}\right) \geq P_{d}^{\min }
$$

where $\mathrm{P}_{\mathrm{f}}\left(\tau_{i}\right)$ measures the amount of the loose of communication opportunities and, hence, it is a measure of the sensing efficiency, and therefore, it must maintained low as much as possible.

By assuming that the outages of the channels that deliver the data from the consumers to RDM are statistically independent ${ }^{2}$ each other, the overall channel-outage probability is given by:

$$
P_{o g}=1-\prod_{i=1}^{N}\left[\left(1-P_{o g}\left(\tau_{i}\right)\right]\right.
$$

In the following we assume that the sensing procedures adopt the energy-detection devices. Such an assumption is largely considered in the literature [14] since the energy detector exhibits the lowest complexity, requires as a-priory knowledge only to know where the spectral signal bandwidths are located and, moreover, it is almost robust against the interference scenario. The energy detector integrates the square of the signal in the bandwidth $\mathrm{W}$ over the sensing time $\tau_{\mathrm{i}}$ and compares the resulting value, namely the energy, with a threshold $\varepsilon_{\mathrm{i}}$ to decide whether the $\mathrm{Ch}_{\mathrm{c}}(\mathrm{i})$ is occupied by PU or is idle. In [9] it is shown that the collision probability in presence of the cognitive-based switching procedure is lower than that when such a procedure is not performed.

According to this assumption the detection probability and the false-alarm probability can be expressed in terms of Qfunction as:

$$
\begin{gathered}
P_{d}\left(\tau_{i}\right)=Q\left[\sqrt{W \tau_{i}}\left(\frac{\varepsilon_{i}}{2 W \tau_{i}\left(\gamma_{i}+1\right)}-1\right)\right] \\
P_{f}\left(\tau_{i}\right)=Q\left[\sqrt{W \tau_{i}}\left(\frac{\varepsilon_{i}}{2 W \tau_{i}}-1\right)\right]
\end{gathered}
$$

where $\gamma_{\mathrm{i}}$ is the signal-to-noise ratio (SNR) at the i-th gateway transceiver, $\varepsilon_{\mathrm{i}}$ is the threshold of the test, normalized to $\sigma_{\mathrm{i}}{ }^{2}$ which is the mean square of the additive white Gaussian noise and

$$
Q(x) \triangleq \int_{x}^{\infty} \frac{1}{2 \pi} e^{-\frac{\alpha^{2}}{2 \pi}} d \alpha
$$

is the Q-function.

To guarantee the minimum interference level $\mathrm{P}_{\mathrm{d}}{ }^{\text {min }}$ against the PU the threshold $\varepsilon_{\mathrm{i}}$ from (2.5) it must be set as follows:

$$
\varepsilon_{i}=2 W \tau_{i}\left[Q^{-1}\left(P_{d}^{\min }\right)+\sqrt{W \tau_{i}}\right]\left(\gamma_{i}+1\right)
$$

By substituting (2.8) into (2.6) one has:

$$
\begin{gathered}
P_{f}\left(\tau_{i}\right)=Q\left[\gamma_{i} \sqrt{W \tau_{i}}+Q^{-1}\left(P_{d}^{\min }\right)\left(\gamma_{i}+1\right)\right] \\
P_{f \min }(i)=Q\left[\gamma_{i} \sqrt{W T}+Q^{-1}\left(P_{d}^{\min }\right)\left(\gamma_{i}+1\right)\right]
\end{gathered}
$$

By accounting for the decreasing property of the Q-function it results, as expected, that the $\mathrm{P}_{\mathrm{f}}(\mathrm{i})$ decreases as the sensing time $\tau_{\mathrm{i}}$ increases.

The switching probability can be re-expressed by setting $\mathrm{P}_{\mathrm{d}}(\mathrm{i})$ equal to $\mathrm{P}_{\mathrm{d}}{ }^{\mathrm{min}}$ and by substituting (2.9) in (2.1):

$$
\begin{aligned}
P_{s w}\left(\tau_{i}\right) & =1-P_{1}(i) P_{d}^{\min }- \\
& -P_{0}(i) Q\left[\gamma_{i} \sqrt{W \tau_{i}}+Q^{-1}\left(P_{d}^{\min }\right)\left(\gamma_{i}+1\right)\right] \\
P_{s w}^{\min }(i) & =1-P_{1}(i) P_{d}^{\min }- \\
& -P_{0}(i) Q\left[\gamma_{i} \sqrt{W T}+Q^{-1}\left(P_{d}^{\min }\right)\left(\gamma_{i}+1\right)\right]
\end{aligned}
$$

Moreover, by substituting (2.10) in (2.2) one has:

$$
\begin{aligned}
P_{o g}\left(\tau_{i}\right) & =\left[\mathrm{P}\left(C h_{c}(\mathrm{i})\right)-\mathrm{P}\left(C h_{u l}(\mathrm{i})\right]\right. \\
& \cdot Q\left\{\gamma_{i} \sqrt{W \tau_{i}}+Q^{-1}\left(P_{d}^{\text {min }}\right)\left(\gamma_{i}+1\right)\right\}+ \\
& +\left[\mathrm{P}\left(C h_{c}(\mathrm{i})\right)-\mathrm{P}\left(C h_{u l}(\mathrm{i})\right] P_{1}(i) P_{d}^{\text {min }}+\right. \\
& +\mathrm{P}\left(C h_{u l}(i)\right)
\end{aligned}
$$

From (2.10) and (2.11), one has that, as $\tau_{i}$ increases and therefore, the sensing accuracy improves, $P_{s w}\left(\tau_{i}\right)$ tends to its maximum value $1-P_{1}(i) P_{d}^{\min }$ and the channel-outage $P_{o g}\left(\tau_{i}\right)$ (2.2) tends to its minimum value:

$$
\begin{aligned}
P_{o u}^{\min } & =\left[\mathrm{P}\left(C h_{c}(\mathrm{i})\right)-\mathrm{P}\left(C h_{u l}(\mathrm{i})\right] P_{s w}^{\min }+\right. \\
& +\mathrm{P}\left[C h_{u l}(\mathrm{i})\right]
\end{aligned}
$$

On the other hand, the increasing of the sensing time gives less time for transmitting since the sensing rate, say $1 / \mathrm{T}$, is fixed to allow an adequate monitoring of the PU traffic activity, and therefore the transmitting time

$$
T_{x_{i}}=T-\tau_{i}
$$

decreases. Such a consideration suggests that the optimization of the only overall outage (2.4) is not a right choice but the cost function has to take into account also the trade-off between the advantages gained by the increasing of the 
sensing accuracy with the sensing time increasing and the decreasing of the transmission capacity which depends on the transmission times $T_{x_{i}}(i=1,2, \ldots N)$

The sensing time can be single out, therefore, according to this maximization procedure:

$$
\begin{aligned}
& \left(\tau_{1}, \tau_{2}, \ldots, \tau_{N}\right)^{o p t}= \\
& \quad=\arg \max _{\tau_{1}, \tau_{2}, \ldots, \tau_{N}}\left(1-P_{o g}\left(\tau_{1}, \tau_{2}, \ldots, \tau_{N,}\right)+\right. \\
& \left.\quad+\frac{\sum_{i=1}^{N} R\left(\tau_{i}, \gamma_{i}\right)}{R_{\max }}\right)= \\
& \quad=\arg \max _{\tau_{1}, \tau_{2}, \ldots, \tau_{N}}\left[\prod _ { i = 1 } ^ { N } \left[\left(1-P_{o g}\left(i, \tau_{i}\right)\right]+\right.\right. \\
& \left.\quad \frac{\sum_{i=1}^{N} R\left(\tau_{i}, \gamma_{i}\right)}{R_{\max }}\right]
\end{aligned}
$$

where:

$$
R\left(\tau_{i}, \gamma_{i}\right)=C_{i} \frac{T-\tau_{i}}{T}
$$

and

$$
R_{\max _{i}}=C_{i}
$$

with $C_{i}$ denoting the bit rate of the $\mathrm{Ch}(\mathrm{i})$ defined as:

$$
C h(i)=P_{s w}(i) C_{c_{i}}+\left(1-P_{s w}(i)\right) C_{u l_{i}}
$$

where $C_{c_{i}}$ and $C_{u l_{i}}$ are the bit rate of the ith cognitive channel and unlicensed channel, respectively.

In the particular case of $\gamma_{i}=\gamma(i=1,2, \ldots N), P_{1}(i)=P_{1}$ and $C_{i}=$ $\mathrm{C}$ the optimization problem (2.14) becomes more simple in that $\tau_{1}=\tau_{2}=\cdots \tau_{N}=\tau$

$$
\tau^{\text {opt }}=\arg \max _{\tau}\left[\left[\left(1-P_{\text {og }}(i, \tau)\right]^{N}+\frac{R(\tau, \gamma)}{R_{\max }}\right]\right.
$$

where $P_{o g}(i, \tau)$ is given by $(2.2)$.

\section{NUMERICAL RESULTS}

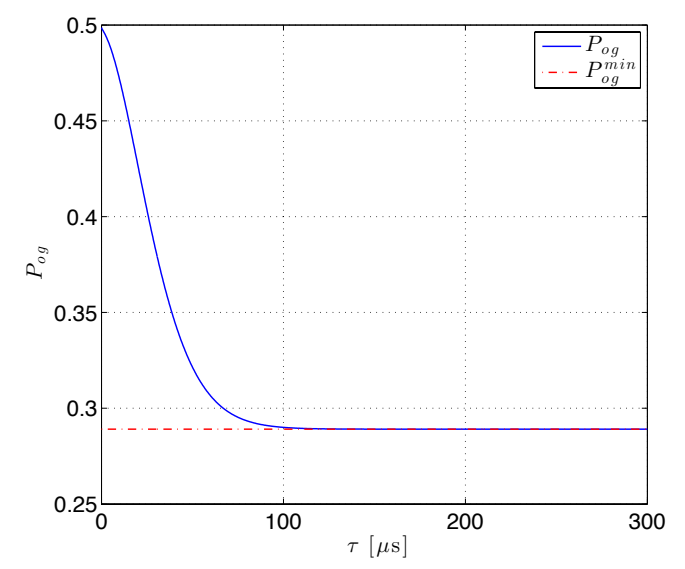

Fig. 3 Probability of Channel Outage VS Sensing Time
In this section we report results derived by the analytical analysis reported in the previous section. More precisely, Fig. 3 shows the overall probability of channel outage $P_{o g}(i)(2.11)$ versus the common sensing time $\tau$ for fixed values of the bandwidth $\mathrm{W}=6 \mathrm{Mz}$, the SNR $\gamma=-15 d B$, the sensing period $\mathrm{T}=0.3 \mathrm{msec}$, the probability $\mathrm{P}_{1}(\mathrm{i})=0.1$, the channel outage $\mathrm{P}\left(\mathrm{Ch}_{\mathrm{c}}(\mathrm{i})\right)=0.2$ and $\mathrm{P}\left(\mathrm{Ch}_{\mathrm{ul}}(\mathrm{i})\right)=0.5, C_{c_{i}}=10, C_{u l_{i}}=5$. As expected $P_{o g}(i)$ decreases with $\tau$ and reaches its minimum value (2.12) asymptotically as $\tau \rightarrow \infty$. The behaviour of $P_{o g}(i)$ with varying values of $\gamma$ has been also analysed: as $\gamma$ decreases such a minimum value is approached for greater values of $\tau$.

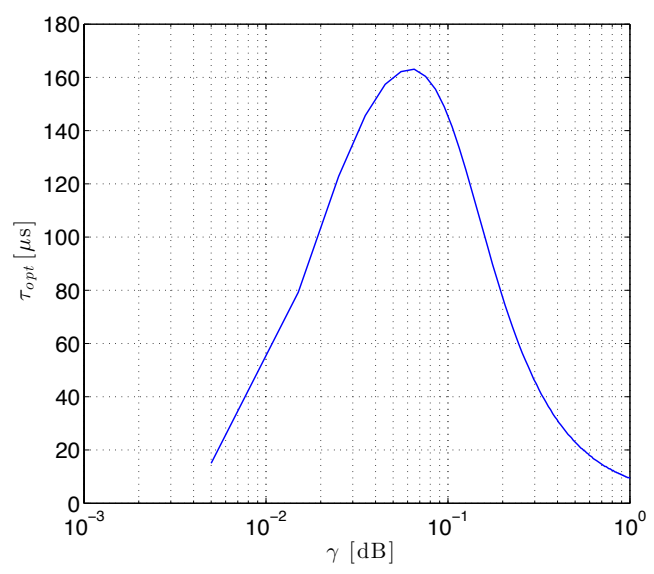

Fig. 4 Optimum Vale of $\tau$ VS $\gamma$

Fig. 4 reports the optimum value of $\tau$ (2.18) as a function of $\gamma$ : such a value increases with $\gamma$ up to reach a maximum value and, then this optimum value decreases with $\gamma$. This behaviour can justified as follows: for smaller values of $\gamma$ the optimum sensing time increases since the benefit gained by the sensing accuracy (the first term in (2.18)) dominates with respect the second term whereas, when the value of $\gamma$ becomes larger the optimum sensing time $\tau^{\text {opt }}$ decreases since the sensing accuracy benefit saturates and smaller values of $\tau^{o p t}$ allow the second term of (2.18) to dominate.

\section{CONCLUSIONS}

The paper deals with the reduction of the channel outage in a smart grid scenario since it plays a crucial role in the DRM control management performances. A study on a two-way cognitive-based switching procedure is carried on and the tools for the optimum sensing time evaluation are provided. Such evaluation considers a cost function that takes into account both the sensing-accuracy improvement gained by increasing the sensing time and the transmission capacity degradation induced by increasing the sensing time.

\section{REFERENCES}

[1] Q. Li, Z. Feng, W. Li, A. Gulliver, P. Zhang “ Joint Spatial and Temporal Spectrum Sharing for Demand Response Management in cognitive Radio Enabled Smart Grid," IEEE Trans. on Smart Grid, vol. 5, no. 4, pp. 1993-2001, July 2014. 
[2] R. Yo, Y. Zhang, C. Yuen, S. Xie, M. Guizani, “ Cognitive Radio Based Hierarchical Communications Infrastructure for Smart Grid," IEEE Network, pp.6-14, September/October 2011.

[3] I A. Metke, and R. Ekl, " Security Technology for Smart Grid Networks," IEEE Trans. on Smart Grid, vol. 1, no. 1, pp. 99-107, January 2010.

[4] "Report of the Spectrum Efficiency Working Group, Federal Communications," Federal Communications, Spectrum Policy Task Force, Tech. Rep., 2002.

[5] S. Haykin, "Cognitive Radio: Brain-empowered wireless communications," IEEE J. Sel. Areas Commun., vol.23, no.2, pp. 201220, Feb. 2005.

[6] B. Wang and K. J. Liu, " Advance in cognitive radio networks: A Survey,” IEEE J. Sel. Areas Commun., vol. 5, no. 1, pp.5-23, Jan. 2011.

[7] M.G. Khoshkolgh, K. Navaie, and H. Yanikomeroglu, "Access Strategies for spectrum sharing in fading environment: Overlay, underlay and mixed," IEEE Trans. Mobile Computer., vol. 9, no.12 , pp. 1780-1793, Dec. 2010.

[8] R. Deng, J. Chen, X. Cao, Y. Zhang, S. Maharian, and S. Gjessing, "Sensing-Performance Tradeoff in Cognitive Radio Enabled Smart Grid," IEEE Trans. on Smart Grid, vol. 4, no. 1, pp. 302-310, March 2013.

[9] J. Huang, H. Wang, Y. Qian, and C. Wang, "Priority-Based Traffic Scheduling and Utility Optimization for Cognitive Radio
Communication Infrastructure-Based Smart Grid, " IEEE Trans. on Smart Grid, vol. 4, no. 1, pp. 78-86, March 2013.

[10] A. S. Cacciapuoti, M. Caleffi, L. Paura, R. Savoia, "Decision Maker Approaches for Cooperative Spectrum Sensing: Participate or Not Participate in Sensing?", IEEE Transactions on Wireless Communications, Vol. 12, Issue 5, Maggio 2013.

[11] A.S. Cacciapuoti, M. Caleffi, F. Marino, L. Paura, "Routing update period in cognitive radio ad hoc networks," Proc. of IEEE International Workshop on Measurements and Networking; Naples; Italy; 7 -8 October 2013.

[12] A.S. Cacciapuoti, I.F. Akyldiz, L. Paura, “Optimal primary-user mobility aware spectrum sensing design for cognitive radio networks," IEEE J. Sel. Areas Commun., vol. 31, no. 11, pp.21612172, Nov. 2013.

[13] A.S. Cacciapuoti, M. Caleffi, F. Marino, L. Paura, "Maximizing the Route Capacity in Cognitive Radio Networks", Proc. of IEEE International Conference on Sensing, Communication, and Networking (IEEE SECON), Singapore, June 2014.

[14] T. Yücek and H. Arslan, "A survey of spectrum sensing algorithms for cognitive radio applications ," IEEE Commun. Surveys Tuts., vol. 11, no. 1, pp. 116-130, 2009. 